\title{
On the structure of the Selberg class, IV: basic invariants
}

\author{
by \\ J. Kaczorowski (Poznań) and A. Perelli (Genova)
}

1. Introduction. This paper is a continuation of part II (see [6]) of the series. It is well known that the $\gamma$-factors in the functional equation of a function $F(s)$ in the extended Selberg class $\mathcal{S}^{\sharp}$ (see below for definitions) are uniquely determined by $F(s)$ up to multiplicative constants, although their shape can be considerably changed by means of suitable identities satisfied by the $\Gamma$ function; see Theorem 2.1 of Conrey-Ghosh [1]. Such a property of $\gamma$-factors leads to the notion of invariant (see [6]); we recall here that an invariant (resp. a numerical invariant) of $F \in \mathcal{S}^{\sharp}$ is an expression (resp. a number), defined in terms of the data in the functional equation of $F(s)$, depending only on the function $F(s)$ and not on the form of the functional equation. In [6] we obtained a transformation algorithm for $\gamma$-factors and, as a consequence, we gave a simple criterion characterizing invariants of functions in $\mathcal{S}^{\sharp}$. Amongst other things, such a criterion allowed us to exhibit several interesting new invariants.

In this paper we deal with the following two natural problems about invariants. The first problem is to construct a universal set of numerical invariants characterizing the functional equation; by universal we mean that the choice of such invariants is the same for every $F \in \mathcal{S}^{\sharp}$. Any such set of invariants will be called a set of basic invariants. Note that, given one such set, it is easy to build infinitely many sets of basic invariants. The second problem is to construct a form of the functional equation where all data are invariants, which we call an invariant form. Again, one can easily build infinitely many such forms of the functional equation from a given one. Note that the two problems are closely connected; for instance, a solution of the second problem immediately provides a set of numerical invariants, depending on each $F \in \mathcal{S}^{\sharp}$, characterizing the functional equation of $F(s)$. In this paper we deal first with the problem of constructing a set of basic

2000 Mathematics Subject Classification: Primary 11M41. 
invariants, and then we turn to the second problem by constructing a special type of invariant form, called canonical form, of the functional equation.

We keep as much as possible the notation introduced in [5], [6] and in the survey paper [4]. In particular, we recall that a function $F \in \mathcal{S}^{\sharp}$ satisfies a functional equation of type

$$
\gamma(s) F(s)=\omega \bar{\gamma}(1-s) \bar{F}(1-s),
$$

where $|\omega|=1, \bar{f}(s)=\overline{f(\bar{s})}$ and the $\gamma$-factor $\gamma(s)$ has the form

$$
\gamma(s)=Q^{s} \prod_{j=1}^{r} \Gamma\left(\lambda_{j} s+\mu_{j}\right), \quad Q>0, \lambda_{j}>0, \operatorname{Re} \mu_{j} \geq 0 .
$$

We also recall that degree, $\xi$-invariant, conductor and root number, defined by

$$
\begin{gathered}
d_{F}=2 \sum_{j=1}^{r} \lambda_{j}, \quad \xi_{F}=2 \sum_{j=1}^{r}\left(\mu_{j}-1 / 2\right)=\eta_{F}+i \theta_{F}, \\
q_{F}=(2 \pi)^{d_{F}} Q^{2} \prod_{j=1}^{r} \lambda_{j}^{2 \lambda_{j}}, \quad \omega_{F}^{*}=\omega e^{-i(\pi / 2)\left(\eta_{F}+1\right)}\left(\frac{q_{F}}{(2 \pi)^{d_{F}}}\right)^{i \theta_{F} / d_{F}} \prod_{j=1}^{r} \lambda_{j}^{-2 i \operatorname{Im} \mu_{j}},
\end{gathered}
$$

respectively, are invariants of $F(s)$. We also allow $d_{F}=0$, in which case $\gamma$-factors simply reduce to $\gamma(s)=Q^{s}$. However, in what follows we consider only functions $F \in \mathcal{S}^{\sharp}$ with $d_{F}>0$, since the whole theory of invariants is trivial in the case $d_{F}=0$; see Theorem 1 of [5].

We introduce the following notation. As usual, we denote by $B_{n}(x)$ the $n$th Bernoulli polynomial defined by

$$
\frac{z e^{x z}}{e^{z}-1}=\sum_{n=0}^{\infty} B_{n}(x) \frac{z^{n}}{n !}, \quad|z|<2 \pi,
$$

hence $B_{0}(x)=1, B_{1}(x)=x-1 / 2, B_{2}(x)=x^{2}-x+1 / 6$ and so on. Moreover, for a given $F \in \mathcal{S}^{\sharp}$ we write

$$
K_{F}(z)=z \sum_{j=1}^{r} \frac{e^{z \mu_{j} / \lambda_{j}}}{e^{z / \lambda_{j}}-1}, \quad z \in \mathbb{C}
$$

and

$$
H_{F}(n)=2 \sum_{j=1}^{r} \frac{B_{n}\left(\mu_{j}\right)}{\lambda_{j}^{n-1}}, \quad n=0,1, \ldots
$$

Note that both $K_{F}(z)$ and $H_{F}(n)$ are additive with respect to the function $F(s)$, i.e., for $F, G \in \mathcal{S}^{\sharp}$ we have

$$
K_{F G}(z)=K_{F}(z)+K_{G}(z), \quad H_{F G}(n)=H_{F}(n)+H_{G}(n) .
$$

We start with the construction of a set of basic invariants. 
TheOREM 1. The function $K_{F}(z)$ and the numbers $H_{F}(n), n \geq 0$, are invariants of $F \in \mathcal{S}^{\sharp}$. Moreover, two functions $F, G \in \mathcal{S}^{\sharp}$ satisfy the same functional equation if and only if

(1.1) $\quad q_{F}=q_{G}, \quad \omega_{F}^{*}=\omega_{G}^{*}$ and $H_{F}(n)=H_{G}(n)$ for all $n \geq 0$.

The invariants $H_{F}(n), n \geq 0$, are called the $H$-invariants of $F \in \mathcal{S}^{\sharp}$. Observe that degree and $\xi$-invariant are recovered as

$$
d_{F}=H_{F}(0), \quad \xi_{F}=H_{F}(1) .
$$

Later on, see (2.8) below, we give a meaning to all $H$-invariants in terms of a certain asymptotic expansion; at present we leave open the problem of giving a meaning to the $H_{F}(n)$ in terms of $F(s)$, without explicit reference to the functional equation. In view of Theorem $1, q_{F}, \omega_{F}^{*}$ and the $H$-invariants form a set of basic invariants. In Theorem 3 below, given any fixed $F \in \mathcal{S}^{\sharp}$, we construct a finite set of numerical invariants characterizing the functional equation of $F(s)$.

We remark that the proof of Theorem 1 shows that if $\gamma_{1}(s)$ and $\gamma_{2}(s)$ are $\gamma$-factors of $F(s)$ and $G(s)$, respectively, and (1.1) is replaced by the weaker assumptions

$$
H_{F}(n)=H_{G}(n) \quad \text { for all } n \geq 0
$$

and

$$
q_{F}=q_{G} \quad \text { and } \quad H_{F}(n)=H_{G}(n) \text { for all } n \geq 0,
$$

then we obtain the weaker statements

$$
\gamma_{1}(s)=e^{a s+b} \gamma_{2}(s) \quad \text { with } a=\frac{1}{2} \log \left(q_{F} / q_{G}\right) \text { and some constant } b \in \mathbb{C}
$$
and

$$
\gamma_{1}(s)=c \gamma_{2}(s) \quad \text { for some constant } c \in \mathbb{C},
$$

respectively; see (2.5), (2.7) and (2.9) below. Moreover, the function $K_{F}(z)$ determines all $H$-invariants of $F(s)$ and vice versa, since

$$
K_{F}(z)=\frac{1}{2} \sum_{n=0}^{\infty} \frac{H_{F}(n)}{n !} z^{n} ;
$$

see (2.3) below. Hence $K_{F}(z)$ also determines the $\gamma$-factors of $F(s)$ up to factors $e^{a s+b}$.

In Section 2 we give two proofs of the second part of Theorem 1. The first proof is simpler, while the second also provides the above mentioned interpretation of $\mathrm{H}$-invariants as coefficients of a certain asymptotic expansion.

The function $K_{F}(z)$ gives rise to other interesting invariants. We first recall a few definitions and results from [6]. The $\gamma$-class number $h_{F}$ is the number of $\mathbb{Q}$-equivalence classes arising from the coefficients $\lambda_{1}, \ldots, \lambda_{r}$ of a $\gamma$-factor of $F \in \mathcal{S}^{\sharp}$, and $h_{F}$ is an invariant. $F(s)$ is reduced if it has a $\gamma$-factor 
with $0 \leq \operatorname{Re} \mu_{j}<1$ for $j=1, \ldots, r$. The canonical polynomial $P_{F}(s)$ is the monic polynomial obtained by reducing a $\gamma$-factor $\gamma(s)$ of $F(s)$ by means of the factorial formula $\Gamma(s+1)=s \Gamma(s)$ as

$$
\gamma(s)=c_{\gamma} P_{F}(s) \widetilde{\gamma}(s),
$$

where $c_{\gamma} \in \mathbb{C}$ and $\widetilde{\gamma}(s)$ has $0 \leq \operatorname{Re} \widetilde{\mu}_{j}<1$ for $j=1, \ldots, r$. Moreover, we recall that $P_{F}(s)$ is an invariant, and $F(s)$ is reduced if and only if $P_{F}(s)=1$ identically.

We split a $\gamma$-factor $\gamma(s)$ of $F \in \mathcal{S}^{\sharp}$ according to $\mathbb{Q}$-equivalence classes of the $\lambda_{j}$ 's as

$$
\gamma(s)=Q^{s} \prod_{j=1}^{h_{F}} \gamma_{j}(s)
$$

and, analogously, we split $K_{F}(z)$ as

$$
K_{F}(z)=\sum_{j=1}^{h_{F}} K_{j}(z) .
$$

The functions $K_{j}(z), 1 \leq j \leq h_{F}$, are almost invariants, in the sense that if functions $\widetilde{K}_{j}(z)$ are constructed in the same way as the $K_{j}(z)$ starting from another $\gamma$-factor $\widetilde{\gamma}(s)$ of $F(s)$, then

$$
K_{j}(z)=\widetilde{K}_{j}(z)+z E_{j}(z)
$$

where $E_{j}(z)$ are exponential polynomials (see Section 3 ). We define the canonical exponents $\Lambda_{1}, \ldots, \Lambda_{h_{F}}$ of $F(s)$ by

$$
\Lambda_{j}=\max \left\{\Lambda \in \mathbb{R}:\left(e^{z / \Lambda}-1\right) K_{j}(z) \text { is entire }\right\} .
$$

The canonical exponents exist, are positive and distinct, and of course are invariants by (1.6); see Lemma 3.2 below. We remark that the difficulty is to show that the functions $K_{j}(z)$ are not entire.

We say that an expression of the form

$$
\gamma(s)=e^{a s+b} \prod_{j=1}^{h_{F}} \prod_{k=1}^{M_{j}} \Gamma\left(\lambda_{j} s+\mu_{j, k}\right),
$$

with $a \in \mathbb{R}, b \in \mathbb{C}, \lambda_{j}>0$ and $\operatorname{Re} \mu_{j, k} \geq 0$, is a balanced form of the $\gamma$-factor $\gamma(s)$ if all ratios $\Lambda_{j} / \lambda_{j}, 1 \leq j \leq h_{F}$, are equal. In such a case, the common ratio $\Lambda_{j} / \lambda_{j}$ is called the reduction factor. Every $\gamma$-factor has a balanced form, and the reduction factor of any balanced form is a positive integer; see Lemma 3.3 below. We define the reduction index $l_{F}$ of $F \in \mathcal{S}^{\sharp}$ as the minimal reduction factor of the balanced forms of $\gamma$-factors of $F(s)$. Clearly, the positive integer $l_{F}$ is an invariant of $F(s)$.

Our motivation for introducing the notion of balanced form comes from the fact that the functional equations of all $L$-functions known to us have 
$\gamma$-factors either in balanced form or easily transformable to balanced form. More precisely, such $L$-functions $F(s)$ have $h_{F}=1$ and a $\gamma$-factor with all $\lambda_{j}=1 / 2$; in [6] we conjectured that this is in fact the case for all functions in $\mathcal{S}^{\sharp}$.

Canonical exponents and reduction index can be used to obtain an invariant form of the functional equation where, in addition, the $\gamma$-factor is in balanced form. In fact, writing

$$
\begin{aligned}
& Q_{F}=\left(q_{F}(2 \pi)^{-d_{F}} l_{F}^{d_{F}} \prod_{j=1}^{h_{F}} \Lambda_{j}^{-2 K_{j} \Lambda_{j}}\right)^{1 / 2}, \\
& \omega_{F}=\omega_{F}^{*} e^{i(\pi / 2)\left(\eta_{F}+1\right)}\left(\frac{q_{F}}{(2 \pi)^{d_{F}}}\right)^{-i \theta_{F} / d_{F}} l_{F}^{-i \theta_{F}} \prod_{j=1}^{h_{F}} \prod_{k=1}^{l_{F} K_{j}} \Lambda_{j}^{2 i \operatorname{Im} \mu_{j, k}},
\end{aligned}
$$

we have

Theorem 2. Every $F \in \mathcal{S}^{\sharp}$ uniquely determines positive integers $K_{j}$ $\left(1 \leq j \leq h_{F}\right)$ such that

$$
\gamma_{0}(s) F(s)=\omega_{F} \bar{\gamma}_{0}(1-s) \bar{F}(1-s)
$$

where

$$
\gamma_{0}(s)=Q_{F}^{s} \prod_{j=1}^{h_{F}} \prod_{k=1}^{l_{F} K_{j}} \Gamma\left(\frac{\Lambda_{j}}{l_{F}} s+\mu_{j, k}\right),
$$

the $\mu_{j, k}$ 's $\left(1 \leq j \leq h_{F}, 1 \leq k \leq l_{F} K_{j}\right)$ are uniquely determined $(\bmod \mathbb{Z})$ complex numbers with $\operatorname{Re} \mu_{j, k} \geq 0$, and $Q_{F}, \omega_{F}$ are given by (1.8). Moreover, the $\mu_{j, k}$ 's are uniquely determined if $h_{F}=1$ or if $F(s)$ is reduced, and $l_{F}=1$ in the latter case.

Note that functional equation (1.9) has $\gamma$-factor in balanced form, but it is not necessarily in invariant form. In the cases where all data of (1.9) are invariants, i.e., when the $\mu_{j, k}$ 's are uniquely determined, we say that (1.9) is the canonical form of the functional equation of $F(s)$. In this case $\gamma_{0}(s)$ is denoted by $\gamma_{F}(s)$. We recall from [6] that, conjecturally, $h_{F}=1$ for every $F \in \mathcal{S}^{\sharp}$, and hence we expect that the canonical form exists for every $F \in \mathcal{S}^{\sharp}$.

We remark that the non-uniqueness of the $\mu_{j, k}$ 's can be eliminated at the cost of the explicit presence of the canonical polynomial in the functional equation. In fact, the proof of Theorem 2 (see (3.13) below) shows that every $F \in \mathcal{S}^{\sharp}$ satisfies the functional equation

$$
\begin{gathered}
\gamma_{1}(s) F(s)=\omega_{1} \bar{\gamma}_{1}(1-s) \bar{F}(1-s), \\
\gamma_{1}(s)=Q_{1}^{s} P_{F}(s) \prod_{j=1}^{h_{F}} \prod_{h=1}^{K_{j}} \Gamma\left(\Lambda_{j} s+\widetilde{\mu}_{j, h}\right)
\end{gathered}
$$


with $0 \leq \operatorname{Re} \widetilde{\mu}_{j, h}<1$. In this case the $\widetilde{\mu}_{j, h}$ 's are uniquely determined, and $Q_{1}$ and $\omega_{1}$ have the expressions of $Q_{F}$ and $\omega_{F}$ in (1.8), respectively, with $l_{F}=1$ and $\mu_{j, k}$ replaced by $\widetilde{\mu}_{j, h}$. Although (1.10) is not a priori of the type allowed by the axioms of the Selberg class, it can be transformed into such a functional equation, by absorbing $P_{F}(s)$ into suitable $\Gamma$-factors. In particular, (1.10) can be transformed into the canonical form, when it exists.

From the definition of $l_{F}$ we see that (1.9) has the minimum number of $\Gamma$-factors amongst the $\gamma$-factors of $F(s)$ in balanced form. Observe that if $h_{F}=1$ then a $\gamma$-factor is in balanced form provided its $\lambda$-coefficients are all equal. Therefore, if $h_{F}=1$ (which we believe to be the general case in $\mathcal{S}^{\sharp}$ ) the canonical form exists and has the minimum number of $\Gamma$-factors amongst the $\gamma$-factors in balanced form.

The proof of Theorem 2 shows that the data $h_{F}, l_{F}, \Lambda_{j}$ 's, $K_{j}$ 's and $\mu_{j, k}$ 's in the canonical form are determined by $K_{F}(s)$. Hence, in view of (1.3), Theorem 2 provides a constructive proof of Theorem 1, of course in those cases where the canonical form exists.

In Section 3, after the proof of Theorem 2, we outline an algorithm for the computation of (1.9) starting from any given form of the functional equation of $F \in \mathcal{S}^{\sharp}$. For example, the standard functional equations of $\zeta(s)$ and $L(s, \chi), \chi$ primitive Dirichlet character, are already in canonical form, while for the Dedekind zeta function associated with a number field $K$ with $[K: \mathbb{Q}]=n=r_{1}+2 r_{2}$ we have

$$
\begin{aligned}
& h_{F}=1, \quad l_{F}=1, \quad \Lambda_{1}=1 / 2, \quad K_{1}=n, \\
& \mu_{1, k}= \begin{cases}0 & \text { for } 1 \leq k \leq r_{1}+r_{2}, \\
1 / 2 & \text { for } r_{1}+r_{2}+1 \leq k \leq n .\end{cases}
\end{aligned}
$$

Therefore, the canonical form is different from the standard one in this case. Similar computations can be easily performed in the holomorphic modular case as well, thus showing that, of course after normalization to meet the axioms of the Selberg class, the standard functional equation coincides with the canonical form in this case.

In Theorem 1 we proved that conductor, root number and $H$-invariants form a set of basic invariants. Now we show that for any given $F \in \mathcal{S}^{\sharp}$ there exists a finite set of numerical invariants, depending on $F(s)$, characterizing the functional equation of $F(s)$. With the notation in Theorem 2 we write

$$
r_{F}=l_{F} \sum_{j=1}^{h_{F}} K_{j}, \quad n_{F}=2^{2 h_{F}-1} r_{F}-2 h_{F}+1 .
$$

Moreover, we recall from [6] the strong $\boldsymbol{\lambda}$ conjecture asserting that every $F \in \mathcal{S}^{\sharp}$ has a $\gamma$-factor with $\lambda$-coefficients all equal to $1 / 2$; see also Section 9 of [4]. We have 
TheOREM 3. (i) The functional equation of $F \in \mathcal{S}^{\sharp}$ is characterized by the invariants $h_{F}, r_{F}$,

$$
q_{F}, \quad \omega_{F}^{*} \quad \text { and } \quad H_{F}(n) \text { with } n \leq n_{F} .
$$

(ii) Assuming the strong $\boldsymbol{\lambda}$ conjecture, the functional equation of $F \in \mathcal{S}^{\sharp}$ is characterized by the invariants in (1.11) with $n_{F}$ replaced by $d_{F}$. Moreover, this is best possible in the sense that for every integer $d \geq 1$ there exist $F, G \in \mathcal{S}_{d}^{\sharp}$ for which the invariants in (1.11) with $n_{F}$ replaced by $d-1$ are equal, but $F(s)$ and $G(s)$ satisfy different functional equations.

As a consequence of Theorem 3(ii), given any $F \in \mathcal{S}_{d}^{\sharp}$ we expect that $q_{F}$, $\omega_{F}^{*}$ and the $H$-invariants with $n \leq d$ characterize the functional equation of $F(s)$. We remark that in [5] we proved that the functional equation of $F \in \mathcal{S}_{1}^{\sharp}$ is characterized by the triplet $\left(q_{F}, \omega_{F}^{*}, \xi_{F}\right)$. In view of $(1.2)$, this confirms the above expectation in the case $d=1$.

We finally remark that it will be clear from the next sections that none of the proofs in this paper relies in any way on the assumption that the functional equations under consideration have solutions in $\mathcal{S}^{\sharp}$. Therefore, a completely similar set of results can be developed by considering only functional equations, irrespective of whether they have solutions in $\mathcal{S}^{\sharp}$ or not. This is basically a matter of taste, and our choice of referring to functions in $\mathcal{S}^{\sharp}$ comes from the view that $L$-functions are the main concern in our investigations.

Acknowledgments. We wish to thank the referee for his very careful reading of our manuscript and for many suggestions on both improvements of the presentation and simplifications in the proofs. This research was partially supported by Istituto Nazionale di Alta Matematica, by KBN grant 2 PO3A 02417 and by a MURST grant. The authors wish to thank these institutions for their generous support.

2. Proof of Theorem 1. We start with a uniqueness principle for generalized Dirichlet series with complex exponents, of type

$$
f(z)=\sum_{n=1}^{\infty} a_{n} e^{\varrho_{n} z}
$$

with $\varrho_{n}=\alpha_{n}+i \beta_{n}, a_{n} \in \mathbb{C}, \varrho_{n} \neq \varrho_{m}$ if $n \neq m$ and $0 \leq \alpha_{1} \leq \ldots \leq \alpha_{n} \rightarrow \infty$. We remark that, in general, the analogue of the standard uniqueness principle for ordinary Dirichlet series fails in the case of generalized Dirichlet series. A counterexample is given in Section 1.3 of Chapter II of Leontiev's book [7]. However, such a uniqueness principle holds if the series is absolutely convergent on a sufficiently large domain. 
Lemma 2.1. Let $f(z)$ be as in (2.1) and absolutely convergent for $z=$ $x \leq x_{0}$. If $f(z)=0$ for $z=x \leq x_{0}$, then $a_{n}=0$ for every $n \in \mathbb{N}$.

Proof. By contradiction, let $n_{0}$ be the smallest $n$ such that $a_{n} \neq 0$. Moreover, let $m_{0}$ be the largest $n$ such that $\alpha_{n}=\alpha_{n_{0}}$. We may assume without loss of generality that $\alpha_{n_{0}}=0$. Hence for $x \rightarrow-\infty$ we have

$$
0=f(x)=\sum_{n=n_{0}}^{m_{0}} a_{n} e^{\varrho_{n} x}+\sum_{n=m_{0}+1}^{\infty} a_{n} e^{\varrho_{n} x}=\sum_{n=n_{0}}^{m_{0}} a_{n} e^{i \beta_{n} x}+o(1) .
$$

From (2.2) we have

$$
\sum_{n=n_{0}}^{m_{0}}\left|a_{n}\right|^{2}=\lim _{X \rightarrow \infty} \frac{1}{X} \int_{-2 X}^{-X}\left|\sum_{n=n_{0}}^{m_{0}} a_{n} e^{i \beta_{n} x}\right|^{2} d x=0
$$

a contradiction.

Let $\gamma(s)$ be a $\gamma$-factor of $F \in \mathcal{S}^{\sharp},|z|<2 \pi \min _{j=1, \ldots, r}\left|\lambda_{j}\right|$ and $z_{j}=z / \lambda_{j}$. From the definitions of $K_{F}(z), H_{F}(n)$ and Bernoulli polynomials we have

$$
\begin{aligned}
2 K_{F}(z) & =2 \sum_{j=1}^{r} \lambda_{j} \frac{z_{j} e^{\mu_{j} z_{j}}}{e^{z_{j}}-1} \\
& =2 \sum_{j=1}^{r} \lambda_{j} \sum_{n=0}^{\infty} B_{n}\left(\mu_{j}\right) \frac{z_{j}^{n}}{n !}=\sum_{n=0}^{\infty} \frac{H_{F}(n)}{n !} z^{n} .
\end{aligned}
$$

Since a power series uniquely determines its Taylor coefficients, from (2.3) we see that the invariance of the $H_{F}(n), n \geq 0$, follows from the invariance of $K_{F}(z)$.

Now we observe that the poles of $\gamma(s)$ are at $s=-\left(\mu_{j}+k\right) / \lambda_{j}, j=$ $1, \ldots, r$ and $k=0,1, \ldots$, therefore for $\operatorname{Re} z<0$ we have

$$
K_{F}(z)=-z \sum_{j=1}^{r} \sum_{k=0}^{\infty} e^{\frac{\mu_{j}+k}{\lambda_{j}} z}=-z \sum_{\varrho} e^{-\varrho z}
$$

where $\varrho$ runs over the poles of $\gamma(s)$. Hence $K_{F}(z)$ is an invariant of $F \in \mathcal{S}^{\sharp}$, since the poles of a $\gamma$-factor of $F(s)$ are uniquely determined by $F(s)$ itself.

Let $F, G \in \mathcal{S}^{\sharp}$ have $\gamma$-factors $\gamma_{1}(s)$ and $\gamma_{2}(s)$, respectively. In order to prove the second part of Theorem 1 we first assume that $F(s)$ and $G(s)$ have the same $H$-invariants and observe that (2.3), (2.4) and Lemma 2.1 imply that $\gamma_{1}(s)$ and $\gamma_{2}(s)$ have the same poles. Therefore $\gamma_{1}(s) / \gamma_{2}(s)$ is an entire function of order 1 without zeros, and hence

$$
\gamma_{1}(s)=e^{a s+b} \gamma_{2}(s), \quad a, b \in \mathbb{C} .
$$

Moreover, using Stirling's formula with fixed $\sigma$ and $t \rightarrow \pm \infty$ we see that in 
fact

$$
\gamma_{1}(s)=e^{a s+b} \gamma_{2}(s), \quad a \in \mathbb{R}, b \in \mathbb{C} .
$$

Assume now in addition that $F(s)$ and $G(s)$ have the same conductor. With obvious notation, inserting (2.5) in the functional equation of $F(s)$ we see that $F(s)$ also satisfies the functional equation

$$
e^{a s} \gamma_{2}(s) F(s)=\omega_{1} e^{\bar{b}} e^{-b} e^{a(1-s)} \bar{\gamma}_{2}(1-s) \bar{F}(1-s) .
$$

Hence, recalling (1.2) and denoting by $d$ the common degree of $F(s)$ and $G(s)$, computing the conductor of $F(s)$ by means of (2.6) and equating with the conductor of $G(s)$ we obtain

$$
(2 \pi)^{d} e^{2 a} Q_{2}^{2} \prod_{j=1}^{r_{2}} \lambda_{j, 2}^{2 \lambda_{j, 2}}=(2 \pi)^{d} Q_{2}^{2} \prod_{j=1}^{r_{2}} \lambda_{j, 2}^{2 \lambda_{j, 2}} .
$$

Therefore $a=0$ and hence

$$
\gamma_{1}(s)=c \gamma_{2}(s), \quad c \in \mathbb{C} .
$$

Finally, assuming in addition that $F(s)$ and $G(s)$ have the same root number, arguing as above we can show that the constant $c$ in (2.7) is real, and hence $F(s)$ and $G(s)$ satisfy the same functional equation. Theorem 1 is therefore proved, since it is obvious that (1.1) holds if $F(s)$ and $G(s)$ satisfy the same functional equation.

Now we give an interpretation of the $H$-invariants as coefficients of a certain asymptotic expansion. Such an analysis provides another proof of the second part of Theorem 1.

Let $F \in \mathcal{S}^{\sharp}$ and $\gamma(s)$ be a $\gamma$-factor of $F(s)$. From Stirling's asymptotic expansion

$$
\begin{aligned}
\log \Gamma(s+a)= & \left(s+a-\frac{1}{2}\right) \log s-s+\frac{1}{2} \log 2 \pi+\sum_{n=1}^{N}(-1)^{n+1} \frac{B_{n+1}(a)}{n(n+1)} s^{-n} \\
& +O\left(|s|^{-N-1}\right)
\end{aligned}
$$

valid for all $N \in \mathbb{N}, a \in \mathbb{C}$ and $|\arg s|<\pi$ (see Section 1.18 of [2]), we obtain (2.8) $\log \gamma(s)=\frac{1}{2} H_{F}(0) s \log s$

$$
\begin{aligned}
& +\frac{1}{2}\left(\log q_{F}-H_{F}(0) \log 2 \pi e\right) s+\frac{1}{2} H_{F}(1) \log s \\
& +c(\gamma)+\frac{1}{2} \sum_{n=1}^{N} \frac{(-1)^{n+1}}{n(n+1)} H_{F}(n+1) s^{-n}+O\left(|s|^{-N-1}\right)
\end{aligned}
$$

for all $N \in \mathbb{N}$ and $|\arg s|<\pi$, where

$$
c(\gamma)=\sum_{j=1}^{r}\left(\mu_{j}-\frac{1}{2}\right) \log \lambda_{j}+\frac{r}{2} \log 2 \pi .
$$


From (2.8) we see that if $F, G \in \mathcal{S}^{\sharp}$ with $\gamma$-factors $\gamma_{1}(s)$ and $\gamma_{2}(s)$, respectively, have the same $H$-invariants then

$$
\log \frac{\gamma_{1}(s)}{\gamma_{2}(s)}=\frac{1}{2} s \log \frac{q_{F}}{q_{G}}+c\left(\gamma_{1}\right)-c\left(\gamma_{2}\right)+O\left(|s|^{-N}\right)=a s+b+O\left(|s|^{-N}\right),
$$

say, for all $N \in \mathbb{N}$ and $|\arg s|<\pi$. However, (2.9) is not enough for an immediate deduction of the second part of Theorem 1 . To perform such a deduction we proceed as follows.

Starting from the formula

$$
\begin{gathered}
\log \Gamma(s)=\left(s-\frac{1}{2}\right) \log s-s+\frac{1}{2} \log 2 \pi+\int_{0}^{\infty e^{i \beta}} \psi(z) e^{-z s} \frac{d z}{z}, \\
\psi(z)=\frac{1}{e^{z}-1}-\frac{1}{z}+\frac{1}{2}
\end{gathered}
$$

uniformly for $-\pi / 2<\beta<\pi / 2$ and $-(\pi / 2+\beta)<\arg s<\pi / 2-\beta$ (see (5) of Section 1.9 of [2]), we can prove

Lemma 2.2. Let $a \in \mathbb{C}, \delta>0$ and $c \in \mathbb{R}$ sufficiently large be fixed constants. Then, uniformly for $|\arg s| \leq \pi-\delta$ and $|s|>(c / \delta)|a|$, we have $\log \Gamma(s+a)=\left(s+a-\frac{1}{2}\right) \log s-s+\frac{1}{2} \log 2 \pi+\int_{0}^{e^{i \beta}} \psi(z, a) e^{-z s} \frac{d z}{z}+O\left(e^{-\kappa|s|}\right)$, where $\kappa=\kappa(\delta)>0$ is a certain constant, $-\pi / 2<\beta<\pi / 2$ is such that $|\arg s+\beta|<\pi / 2-\delta$ and

$$
\psi(z, a)=\sum_{n=2}^{\infty} \frac{(-1)^{n}}{n !} B_{n}(a) z^{n-1}, \quad|z|<2 \pi .
$$

We omit the proof of Lemma 2.2, which can be obtained by rather long but standard computations along the lines of the proof of Stirling's asymptotic expansion, and only outline the second proof of the second part of Theorem 1.

Assuming that $F, G \in \mathcal{S}^{\sharp}$ have the same $H$-invariants and using Lemma 2.2 instead of Stirling's asymptotic expansion we obtain the following improved version of $(2.9)$ :

$$
\log \frac{\gamma_{1}(s)}{\gamma_{2}(s)}=a s+b+O\left(e^{-\kappa|s|}\right)
$$

uniformly for $|s|$ sufficiently large and $|\arg s| \leq \pi-\delta$, where $\kappa$ and $\delta$ are as in Lemma 2.2. Moreover, writing

$$
f(t)=\frac{\gamma_{1}(1+i t)}{\gamma_{2}(1+i t)} e^{-a(1+i t)-b}-1, \quad t \in \mathbb{R},
$$


and

$$
\begin{aligned}
\mu_{k}(f) & =\int_{-\infty}^{\infty} f(t) t^{k} d t \\
& =\frac{1}{i^{k+1}} \int_{1-i \infty}^{1+i \infty}\left(\frac{\gamma_{1}(s)}{\gamma_{2}(s)} e^{-a s-b}-1\right)(s-1)^{k} d s, \quad k=0,1, \ldots
\end{aligned}
$$

from (2.10) we have

$$
f(t)=O\left(e^{-\kappa|t|}\right) \text { and } \mu_{k}(f)=0 \text { for } k=0,1, \ldots,
$$

the last statement being easily verified by shifting the line of integration in (2.11) to the right and using (2.10).

Consider now the Fourier transform $\widehat{f}(z)$ of $f(t)$. From $(2.12)$ we see that $\widehat{f}(z)$ is holomorphic for $|\operatorname{Im} z|<\kappa /(2 \pi)$ and, moreover,

$$
\widehat{f}^{(k)}(0)=(2 \pi i)^{k} \mu_{k}(f)=0, \quad k=0,1, \ldots
$$

Therefore $\widehat{f}(z)=0$ identically and hence $f(t)=0$ identically. By the definition of $f(t)$, the principle of analytic continuation and (2.9), this gives (2.5) with $a=\frac{1}{2} \log \left(q_{F} / q_{G}\right)$, and the second part of Theorem 1 follows easily.

3. Proof of Theorem 2. We first state the following sharper version of Ritt's [8] theorem on exponential polynomials, due to Shields [9]. In fact, Shields [9] proves a stronger form of Lemma 3.1 below. We recall that an exponential polynomial is an expression of type

$$
f(z)=\sum_{n \leq N} a_{n} e^{\alpha_{n} z}
$$

with $a_{n} \in \mathbb{C}$ and distinct $\alpha_{n} \in \mathbb{C}$.

LEMMA 3.1 (Shields [9]). Let $f(z)$ and $g(z)$ be exponential polynomials and suppose that $f(z) / g(z)$ has only finitely many poles. Then $f(z) / g(z)$ is an exponential polynomial.

Let $\gamma(s)$ be a $\gamma$-factor of $F \in \mathcal{S}^{\sharp}$. We split $\gamma(s)$ and $K_{F}(z)$ into $\mathbb{Q}$ equivalence classes as in (1.4) and (1.5) and, with abuse of notation, write

$$
\gamma_{j}(s)=\prod_{i=1}^{r_{j}} \Gamma\left(\lambda_{i} s+\mu_{i}\right), \quad j=1, \ldots, h_{F} .
$$

It is easy to see that two $\Gamma$-factors belonging to different $\mathbb{Q}$-equivalence classes may have at most one common pole. Therefore, apart from a finite number of elements, the sets of poles of the $\gamma_{j}(s), 1 \leq j \leq h_{F}$, are invariants of $F(s)$. Hence the functions $K_{j}(z)$ are uniquely determined up to addition of an exponential polynomial times $z$, as in (1.6). Thus, the $\Lambda_{j}$ 's defined 
by (1.7) are also invariants, if they exist. The first step in the proof of Theorem 2 is to show that the $\Lambda_{j}$ 's do exist.

Lemma 3.2. Let $F \in \mathcal{S}^{\sharp}$. Then the $\Lambda_{j}$ 's defined by (1.7) exist, are positive and belong to distinct $\mathbb{Q}$-equivalence classes.

Proof. Let $\gamma(s)$ be a $\gamma$-factor of $F(s)$. For $1 \leq j \leq h_{F}$, we recall that $\Lambda_{j}$ denotes the maximal $\Lambda$ such that

$$
\left(e^{z / \Lambda}-1\right) K_{j}(z)
$$

is entire. We start observing that the $\lambda_{i}$ 's in (3.1), $1 \leq i \leq r_{j}$, are all $\mathbb{Q}$-equivalent, and hence we can write

$$
\lambda_{i}=n_{i} \eta_{j}, \quad 1 \leq i \leq r_{j},
$$

with certain positive integers $n_{i}$ and a positive $\eta_{j} \in \mathbb{R}$. Therefore, by the Legendre-Gauss multiplication formula

$$
\Gamma(s)=m^{s-1 / 2}(2 \pi)^{(1-m) / 2} \prod_{k=0}^{m-1} \Gamma\left(\frac{s+k}{m}\right), \quad m=1,2, \ldots,
$$

we rewrite $\gamma_{j}(s)$ in the form

$$
\gamma_{j}(s)=e^{a_{j} s+b_{j}} \prod_{i=1}^{r_{j}^{\prime}} \Gamma\left(\eta_{j} s+c_{i}\right), \quad \operatorname{Re} c_{i} \geq 0
$$

and computing $K_{j}(z)$ using (3.4) we obtain

$$
K_{j}(z)=\frac{z}{e^{z / \eta_{j}}-1} \sum_{i=1}^{r_{j}^{\prime}} e^{c_{i} z / \eta_{j}} .
$$

Hence the set of $\Lambda$ 's such that (3.2) is entire is not empty. Moreover, the poles of $K_{j}(z)$ are contained in the set $\left\{2 \pi i k \eta_{j}: k \in \mathbb{Z}\right\}$.

By (2.4) applied to $K_{j}(z)$, the function $K_{j}(z) / z$ is not an exponential polynomial. Hence, by Lemma $3.1, K_{j}(z)$ has at least one pole, say at $z=$ $2 \pi i k_{0} \eta_{j}$ with some $k_{0}>0$. Such a pole has therefore to be cancelled by a zero of $e^{z / \Lambda}-1$, hence admissible $\Lambda$ 's have the shape

$$
\Lambda=k_{0} \eta_{j} / k, \quad k \in \mathbb{Z} .
$$

Thus $\Lambda \leq k_{0} \eta_{j}$ and the set of admissible $\Lambda^{\prime}$ 's has a maximum, therefore $\Lambda_{j}$ exists for $1 \leq j \leq h_{F}$. Finally, by (3.5) it is clear that the $\Lambda_{j}$ 's are positive and belong to distinct $\mathbb{Q}$-equivalence classes.

For $1 \leq j \leq h_{F}$ let

$$
f_{j}(z)=\left(e^{z / \Lambda_{j}}-1\right) K_{j}(z)
$$


By (2.4) applied to $K_{j}(z)$ we have

$$
K_{j}(z)=-z \sum_{\varrho} e^{-\varrho \Lambda_{j} z / \Lambda_{j}}
$$

where $\varrho$ runs over the poles of $\gamma_{j}(s)$. Hence for $\operatorname{Re} z<0$ we can write

$$
f_{j}(z)=z \sum_{l=1}^{\infty} n_{j}(l) e^{m_{l, j} z / \Lambda_{j}}
$$

with some $n_{j}(l) \in \mathbb{Z}$ and $m_{l, j} \in \mathbb{C}$ satisfying $\operatorname{Re} m_{l, j} \geq 0, \operatorname{Im} m_{l, j} \ll 1$ and

$$
0<A_{j} \leq \frac{\operatorname{Re} m_{l, j}}{l} \leq B_{j}
$$

for $l$ sufficiently large, where $A_{j}, B_{j}, 1 \leq j \leq h_{F}$, are suitable constants. Observe now that $f_{j}(z) / z$ is a quotient of two exponential polynomials and, by definition of the $\Lambda_{j}$ 's, it has at most one pole. Hence by Lemma 3.1 we have $n_{j}(l)=0$ for $l$ sufficiently large. Therefore the sum in (3.7) is finite and, rearranging and repeating terms if necessary, we write (3.7) as

$$
f_{j}(z)=z \sum_{l=1}^{N_{j}} n_{j}(l) e^{m_{l, j} z / \Lambda_{j}}
$$

with $n_{j}(l)=-1$ for $1 \leq l \leq M_{j}$ and $n_{j}(l)=1$ for $M_{j}+1 \leq l \leq N_{j}$. From (3.6) and (3.8) we therefore obtain

$$
K_{j}(z)+z \sum_{l=1}^{M_{j}} \frac{e^{m_{l, j} z / \Lambda_{j}}}{e^{z / \Lambda_{j}}-1}=z \sum_{l=M_{j}+1}^{N_{j}} \frac{e^{m_{l, j} z / \Lambda_{j}}}{e^{z / \Lambda_{j}}-1} .
$$

With the data in (3.9) we build the products

$$
\prod_{l=1}^{M_{j}} \Gamma\left(\Lambda_{j} s+m_{l, j}\right), \quad \prod_{l=M_{j}+1}^{N_{j}} \Gamma\left(\Lambda_{j} s+m_{l, j}\right)
$$

and hence the argument in the proof of Theorem 1 , showing that $K_{F}(z)$ determines $\gamma(s)$ up to a factor $e^{a s+b}$ (see also the remark after Theorem 1), gives in this case the identities

$$
\begin{aligned}
& \gamma_{j}(s) \prod_{l=1}^{M_{j}} \Gamma\left(\Lambda_{j} s+m_{l, j}\right)=e^{a^{(j)} s+b^{(j)}} \prod_{l=M_{j}+1}^{N_{j}} \Gamma\left(\Lambda_{j} s+m_{l, j}\right), \\
& 1 \leq j \leq h_{F},
\end{aligned}
$$

with some $a^{(j)} \in \mathbb{R}$ and $b^{(j)} \in \mathbb{C}$. Observing that for every $1 \leq l \leq M_{j}$ the left hand side of (3.10) has a pole at $s=-m_{l, j} / \Lambda_{j}$, we deduce that there exist an $l^{\prime}=l^{\prime}(l), M_{j}+1 \leq l^{\prime} \leq N_{j}$, and an integer $k_{l, j} \geq 0$ such that

$$
m_{l, j}=m_{l^{\prime}, j}+k_{l, j}, \quad l=1, \ldots, M_{j} .
$$


Therefore, applying $k_{l, j}$ times the factorial formula to $\Gamma\left(\Lambda_{j} s+m_{l, j}\right)$ on the left hand side of (3.10), dividing both sides by the common $\Gamma$-factors obtained in this way, reducing (again by the factorial formula) the remaining $\Gamma$-factors on the right hand side and finally multiplying all identities for $1 \leq j \leq h_{F}$, we end up with an identity of the form

$$
\gamma(s)=e^{a_{1} s+b_{1}} R(s) \prod_{j=1}^{h_{F}} \prod_{h=1}^{K_{j}} \Gamma\left(\Lambda_{j} s+\widetilde{\mu}_{j, h}\right)
$$

where $a_{1} \in \mathbb{R}, b_{1} \in \mathbb{C}, R(s)$ is a rational function and $0 \leq \operatorname{Re} \widetilde{\mu}_{j, h}<1$.

Reducing $\gamma(s)$ we have

$$
\gamma(s)=c_{\gamma} P_{F}(s) \widetilde{\gamma}(s)
$$

where $c_{\gamma} \in \mathbb{C}, P_{F}(s)$ is the canonical polynomial of $F(s)$ and $\widetilde{\gamma}(s)$ is the reduced part of $\gamma(s)$ (see Section 3 of [6]), and hence comparing with (3.11) we obtain

$$
\widetilde{\gamma}(s)=e^{a_{2} s+b_{2}} \frac{R(s)}{P_{F}(s)} \prod_{j=1}^{h_{F}} \prod_{h=1}^{K_{j}} \Gamma\left(\Lambda_{j} s+\widetilde{\mu}_{j, h}\right)
$$

with $a_{2} \in \mathbb{R}$ and $b_{2} \in \mathbb{C}$. Since the $\Gamma$-factors on both sides of (3.12) are reduced, from Lemma 2.3(ii) of [6] we see that

$$
R(s)=c_{1} P_{F}(s)
$$

with $c_{1} \in \mathbb{C}$, and hence (3.11) becomes

$$
\gamma(s)=e^{a_{3} s+b_{3}} P_{F}(s) \prod_{j=1}^{h_{F}} \prod_{h=1}^{K_{j}} \Gamma\left(\Lambda_{j} s+\widetilde{\mu}_{j, h}\right)
$$

with $a_{3} \in \mathbb{R}$ and $b_{3} \in \mathbb{C}$. We remark at this point that $a_{3}$, the $K_{j}$ 's and the $\widetilde{\mu}_{j, h}$ 's in (3.13) are uniquely determined by $F(s)$. In fact, if $\gamma^{\prime}(s)$ is a $\gamma$-factor of $F(s)$ we have $\gamma(s)=c_{2} \gamma^{\prime}(s)$ for some $c_{2} \in \mathbb{C}$, and hence (3.13) leads to an identity of type

$$
\prod_{j=1}^{h_{F}} \prod_{h=1}^{K_{j}} \Gamma\left(\Lambda_{j} s+\widetilde{\mu}_{j, h}\right)=e^{a_{4} s+b_{4}} \prod_{j=1}^{h_{F}} \prod_{h=1}^{K_{j}^{\prime}} \Gamma\left(\Lambda_{j} s+\widetilde{\mu}_{j, h}^{\prime}\right)
$$

with $a_{4} \in \mathbb{R}, b_{4} \in \mathbb{C}$ and $0 \leq \operatorname{Re} \widetilde{\mu}_{j, h}^{\prime}<1$. Our assertion follows then by the argument leading to Lemma 2.3(ii) of [6].

Since the left hand side of (3.13) has no zeros, the zeros of $P_{F}(s)$ must be cancelled by poles of the $\Gamma$-factors. Therefore we can write

$$
P_{F}(s)=c_{3} \prod_{(j, h, l)}\left(\Lambda_{j} s+\tilde{\mu}_{j, h}+l\right),
$$


where $c_{3} \in \mathbb{R}$ and the product is over a certain (possibly empty) finite set of triplets $(j, h, l)$ with $1 \leq j \leq h_{F}, 1 \leq h \leq K_{j}$ and $l=0,1, \ldots$ Observe that the expression of $P_{F}(s)$ in (3.14) is not necessarily unique, since $\Gamma$-factors corresponding to different $\mathbb{Q}$-equivalence classes may have a common pole. Applying the Legendre-Gauss multiplication formula (3.3) with

$$
m=\text { maximum } l \text { in }(3.14)+1
$$

to each $\Gamma$-factor in (3.13), from (3.13) and (3.14) we get

$$
\gamma(s)=e^{a_{5} s+b_{5}} \prod_{(j, h, l)}\left(\frac{\Lambda_{j} s+\widetilde{\mu}_{j, h}+l}{m}\right) \prod_{j=1}^{h_{F}} \prod_{h=1}^{K_{j}} \prod_{l=0}^{m-1} \Gamma\left(\frac{\Lambda_{j} s+\widetilde{\mu}_{j, h}+l}{m}\right)
$$

with $a_{5} \in \mathbb{R}$ and $b_{5} \in \mathbb{C}$. Hence by repeated applications of the factorial formula we can absorb the canonical polynomial into new $\Gamma$-factors as

$$
\gamma(s)=e^{a_{6} s+b_{6}} \prod_{j=1}^{h_{F}} \prod_{k=1}^{m K_{j}} \Gamma\left(\frac{\Lambda_{j}}{m} s+\mu_{j, k}^{\prime}\right)
$$

with $a_{6} \in \mathbb{R}, b_{6} \in \mathbb{C}$ and $\operatorname{Re} \mu_{j, k}^{\prime} \geq 0$.

We have

Lemma 3.3. Let $F \in \mathcal{S}^{\sharp}$. Then every $\gamma$-factor of $F(s)$ has a balanced form. Moreover, the reduction factor of any balanced form is a positive integer.

Proof. The first assertion follows from (3.15). In order to prove the second, let

$$
\gamma(s)=e^{a s+b} \prod_{j=1}^{h_{F}} \prod_{k=1}^{M_{j}} \Gamma\left(\lambda_{j} s+\mu_{j, k}\right)
$$

be a balanced form of $\gamma(s)$. Clearly, the function $\left(e^{z / \lambda_{j}}-1\right) K_{j}(z)$ is entire for $1 \leq j \leq h_{F}$, hence $\lambda_{j} \leq \Lambda_{j}$ and we write

$$
\frac{1}{\lambda_{j}}=\frac{k}{\Lambda_{j}}+\frac{\theta}{\Lambda_{j}}
$$

with a positive integer $k$ and $0 \leq \theta<1$. We therefore have

$$
\left(e^{z / \lambda_{j}}-1\right) K_{j}(z)=\left(e^{k z / \Lambda_{j}}-1\right) e^{\theta z / \Lambda_{j}} K_{j}(z)+\left(e^{\theta z / \Lambda_{j}}-1\right) K_{j}(z),
$$

and the first term on the right hand side is easily seen to be entire. Hence the function $\left(e^{\theta z / \Lambda_{j}}-1\right) K_{j}(z)$ is entire as well, thus $\theta=0$ by the maximality of $\Lambda_{j}$, and Lemma 3.3 follows.

Observing that for $1 \leq j \leq h_{F}$ the contribution of the $\Gamma$-factors in the $j$ th $\mathbb{Q}$-equivalence class to the degree $d_{F}$ of $F(s)$ is an invariant, from 
Lemma 3.3, the definition of $l_{F}$ and (3.15) we get

$$
\gamma(s)=e^{a s+b} \prod_{j=1}^{h_{F}} \prod_{k=1}^{l_{F} K_{j}} \Gamma\left(\frac{\Lambda_{j}}{l_{F}} s+\mu_{j, k}\right)
$$

with $a \in \mathbb{R}, b \in \mathbb{C}$ and $\operatorname{Re} \mu_{j, k} \geq 0$. Moreover, $l_{F}$ is the minimal integer such that $(3.16)$ holds. Note that the $\mu_{j, k}$ 's are uniquely determined $(\bmod \mathbb{Z})$. In fact, if

$$
\gamma(s)=e^{a s+b} \prod_{j=1}^{h_{F}} \prod_{k=1}^{l_{F} K_{j}} \Gamma\left(\frac{\Lambda_{j}}{l_{F}} s+\mu_{j, k}\right)=e^{a^{\prime} s+b^{\prime}} \prod_{j=1}^{h_{F}} \prod_{k=1}^{l_{F} K_{j}} \Gamma\left(\frac{\Lambda_{j}}{l_{F}} s+\mu_{j, k}^{\prime}\right),
$$

comparing poles we see that $-l_{F}\left(\mu_{j, k}+h\right) / \Lambda_{j}$ coincides with $-l_{F}\left(\mu_{j, k}^{\prime}+l\right) / \Lambda_{j}$ for suitable integers $h$ and $l$, therefore the $\mu_{j, k}$ 's are unique $(\bmod \mathbb{Z})$.

Rewriting (3.16) as

$$
\gamma(s)=c \gamma_{0}(s), \quad \gamma_{0}(s)=Q_{F}^{s} \prod_{j=1}^{h_{F}} \prod_{k=1}^{l_{F} K_{j}} \Gamma\left(\frac{\Lambda_{j}}{l_{F}} s+\mu_{j, k}\right),
$$

we see that $\gamma_{0}(s)$ is a $\gamma$-factor of $F(s)$. Moreover, $Q_{F}$ and $\omega_{F}$ are easily computed by means of the definition of conductor and of root number, respectively, thus yielding the first part of Theorem 2 .

Observe that if $F(s)$ is reduced then the canonical polynomial is trivial. Hence Theorem 2 follows already from (3.13), by observing that $l_{F}=1$ in this case. Finally, Theorem 2 follows from (3.17) and Lemma 2.3(i) of [6] if $h_{F}=1$.

The proof of Theorem 2 allows the explicit computation of (1.9) from any given form of the functional equation of $F \in \mathcal{S}^{\sharp}$. A sketch of the steps of such a computation can be extracted from the proof of Theorem 2 as follows.

(i) For $j=1, \ldots, h_{F}$ compute the $\Lambda_{j}$ by means of their definition (1.7).

(ii) For $j=1, \ldots, h_{F}$ compute $f_{j}(z)$ in the form (3.8). Then write (3.10) (without explicit computation of the factor $e^{a^{(j)} s+b^{(j)}}$ ).

(iii) Follow the procedure described between (3.10) and (3.11), thus getting (3.13) (without explicit computation of the factor $\left.e^{a_{3} s+b_{3}}\right)$.

(iv) By means of the multiplication and factorial formulae, determine $l_{F}$ and transform (3.13) into (3.16) (without explicit computation of the factor $\left.e^{a s+b}\right)$.

The form (1.9) of the functional equation is then obtained after computation of $Q_{F}$ and $\omega_{F}$ by means of the expressions given by (1.8). 
We end the section with three examples illustrating Theorem 2 and its proof. First of all, observe that the identity

$$
\Gamma(s / 2) \Gamma(s+1)=2 \Gamma(s / 2+1) \Gamma(s)
$$

shows why the $\lambda$ 's in the canonical form of the functional equation have to be equal in each $\mathbb{Q}$-equivalence class, in order to have uniquely determined $\mu$ 's. Moreover, the identity

$$
s \Gamma(s) \Gamma(\sqrt{2} s)=\Gamma(s+1) \Gamma(\sqrt{2} s)=\frac{1}{\sqrt{2}} \Gamma(s) \Gamma(\sqrt{2} s+1)
$$

shows that the $\mu$ 's in (1.9) are not necessarily unique if $h_{F}>1$ and $F(s)$ is not reduced.

Suppose now that

$$
\gamma(s)=\Gamma\left(\frac{s}{2}\right) \Gamma\left(\frac{s+3}{2}\right) .
$$

In this case $h_{F}=1, \Lambda_{1}=1, K_{1}=1, P_{F}(s)=s+1$ and (3.13) becomes

$$
\gamma(s)=e^{a s+b}(s+1) \Gamma(s),
$$

thus showing the need of a further application of the multiplication formula to $(3.13)$ in order to absorb the canonical polynomial into new $\Gamma$-factors.

4. Proof of Theorem 3. For any fixed $N \in \mathbb{N}$ consider the symmetric polynomials

$$
p_{k}\left(X_{1}, \ldots, X_{N}\right)=\sum_{j=1}^{N} X_{j}^{k}, \quad k \in \mathbb{N} .
$$

We need the following lemma, which is a classical result on symmetric functions based on Newton's formulae (see Section 1.5 of [3]).

Lemma 4.1. (i) If $\boldsymbol{z}=\left(z_{1}, \ldots, z_{N}\right), \boldsymbol{w}=\left(w_{1}, \ldots, w_{N}\right) \in \mathbb{C}^{N}$ satisfy

$$
p_{k}(\boldsymbol{z})=p_{k}(\boldsymbol{w}), \quad k=1, \ldots, N,
$$

then there exists a permutation $\sigma$ such that $z_{j}=w_{\sigma(j)}$ for $j=1, \ldots, N$.

(ii) There exist $\boldsymbol{x}, \boldsymbol{y} \in \mathbb{R}^{N}$ such that

$$
p_{k}(\boldsymbol{x})=p_{k}(\boldsymbol{y}) \quad \text { for } k=1, \ldots, N-1 \quad \text { and } \quad p_{N}(\boldsymbol{x}) \neq p_{N}(\boldsymbol{y}) .
$$

Let $F \in \mathcal{S}^{\sharp}$. In order to prove Theorem 3(i) we observe that by Theorem 1 it is sufficient to show that if $G \in \mathcal{S}^{\sharp}$ has $h_{G}=h_{F}, r_{G}=r_{F}$ and

$$
H_{G}(n)=H_{F}(n) \quad \text { for } n \leq n_{F}\left(=n_{G}\right)
$$

$$
H_{G}(n)=H_{F}(n) \quad \text { for all } n \geq 0 .
$$

Denote by $\Lambda_{1}, \ldots, \Lambda_{h_{F}}$ and $\Lambda_{1}^{\prime}, \ldots, \Lambda_{h_{F}}^{\prime}$ the canonical exponents of $F(s)$ and $G(s)$, respectively. Computing $K_{F}(z)$ and $K_{G}(z)$ by means of the form of 
the functional equation given in Theorem 2 we see that the entire function

$$
f(z)=\frac{1}{z} \prod_{j=1}^{h_{F}}\left(e^{l_{F} z / \Lambda_{j}}-1\right)\left(e^{l_{G} z / \Lambda_{j}^{\prime}}-1\right)\left(K_{F}(z)-K_{G}(z)\right)
$$

can be written in the form

$$
f(z)=\sum_{l=1}^{L} e^{\alpha_{l} z}-\sum_{l=1}^{L} e^{\beta_{l} z}
$$

with $\alpha_{l}, \beta_{l} \in \mathbb{C}$ and

$$
L \leq r_{F} 2^{2 h_{F}-1} .
$$

From (1.3) and (4.1) we see that the coefficients of the Taylor expansion of $K_{F}(z)-K_{G}(z)$ vanish for $n \leq n_{F}$, hence from (4.3) we deduce that $f(z)$ has a zero of order at least $n_{F}+2 h_{F}$ at $z=0$. Therefore

$$
f^{(k)}(0)=0, \quad k \leq n_{F}+2 h_{F}-1
$$

and hence computing $f^{(k)}(0)$ by means of (4.4) we obtain

$$
\sum_{l=1}^{L} \alpha_{l}^{k}=\sum_{l=1}^{L} \beta_{l}^{k}, \quad k \leq n_{F}+2 h_{F}-1 .
$$

From Lemma 4.1(i), (4.5) and (4.6) we see that if

$$
n_{F}=r_{F} 2^{2 h_{F}-1}-2 h_{F}+1
$$

then the $\alpha_{l}$ 's are a permutation of the $\beta_{l}$ 's, therefore $f(z)=0$ identically by (4.4) and hence $K_{F}(z)=K_{G}(z)$ by (4.3). As a consequence, (4.2) holds and hence Theorem $3(\mathrm{i})$ follows.

We turn to the proof of Theorem 3(ii). In this case $F(s)$ and $G(s)$ have a functional equation with exactly $d_{F} \Gamma$-factors with $\lambda$-coefficients all equal to $1 / 2$. If we replace $f(z)$ in (4.3) by

$$
f(z)=\frac{e^{2 z}-1}{z}\left(K_{F}(z)-K_{G}(z)\right),
$$

(4.4) becomes

$$
f(z)=\sum_{l=1}^{d_{F}} e^{\alpha_{l} z}-\sum_{l=1}^{d_{F}} e^{\beta_{l} z} .
$$

If we assume (4.1) with $n_{F}$ replaced by $d_{F}$, the argument leading to (4.6) gives in this case

$$
\sum_{l=1}^{d_{F}} \alpha_{l}^{k}=\sum_{l=1}^{d_{F}} \beta_{l}^{k}, \quad k \leq d_{F}
$$

and the first part of Theorem 3(ii) follows from Lemma 4.1(i), (4.7) and (4.8), as before. 
Now we construct examples of $L$-functions of integer degree $d \geq 1$ satisfying (1.11) with $n_{F}$ replaced by $d-1$ and having different functional equations. For $d=1$ we just consider

$$
F(s)=L(s, \chi), \quad G(s)=L(s+i \theta, \chi)
$$

with a primitive Dirichlet character $\chi(\bmod q), q>1$, and $\theta \neq 0$. Recalling that conductor and root number are also shift-invariants while the $\xi$-invariant $H_{F}(1)$ is not shift-invariant (see Section 4 of [6]), we see that $F(s)$ and $G(s)$ provide the required example for $d=1$.

For $d \geq 2$ we fix an even primitive Dirichlet character $\chi(\bmod q), q>1$, and write

$$
L(s, \boldsymbol{x}, \chi)=\prod_{j=1}^{d} L\left(s+i x_{j}, \chi\right), \quad \boldsymbol{x}=\left(x_{1}, \ldots, x_{d}\right) \in \mathbb{R}^{d} .
$$

By Lemma 4.1(ii) there exist $\boldsymbol{\theta}=\left(\theta_{1}, \ldots, \theta_{d}\right), \boldsymbol{\xi}=\left(\xi_{1}, \ldots, \xi_{d}\right) \in \mathbb{R}^{d}$ such that

$$
\sum_{j=1}^{d} \theta_{j}^{k}=\sum_{j=1}^{d} \xi_{j}^{k} \quad \text { for } k=1, \ldots, d-1 \quad \text { and } \quad \sum_{j=1}^{d} \theta_{j}^{d} \neq \sum_{j=1}^{d} \xi_{j}^{d},
$$

and we consider

$$
F(s)=L(s, \boldsymbol{\theta}, \chi), \quad G(s)=L(s, \boldsymbol{\xi}, \chi) .
$$

Again, $\omega_{F}^{*}=\omega_{G}^{*}$ and $q_{F}=q_{G}=q^{d}$ by shift-invariance, while (4.9) implies that

$$
H_{F}(n)=H_{G}(n) \quad \text { for } n=1, \ldots, d-1 \quad \text { and } \quad H_{F}(d) \neq H_{G}(d) .
$$

In fact, in this case the function $f(z)$ in (4.7) becomes

$$
f(z)=\sum_{j=1}^{d} e^{2 i \theta_{j} z}-\sum_{j=1}^{d} e^{2 i \xi_{j} z}
$$

and hence (4.9) implies that

$$
f^{(k)}(0)=0 \quad \text { for } k=1, \ldots, d-1 \quad \text { and } \quad f^{(d)}(0) \neq 0 .
$$

Therefore $K_{F}(z)-K_{G}(z)$ has a zero of order exactly $d$ at $z=0$, thus (4.10) follows. The proof of Theorem 3 is now complete, since by Theorem 1 the functional equations of $F(s)$ and $G(s)$ are different.

\section{References}

[1] J. B. Conrey and A. Ghosh, On the Selberg class of Dirichlet series: small degrees, Duke Math. J. 72 (1993), 673-693.

[2] A. Erdélyi, W. Magnus, F. Oberhettinger and F. G. Tricomi, Higher Transcendental Functions, Vol. I, McGraw-Hill, 1953. 
[3] L. Gaal, Classical Galois Theory, Amer. Math. Soc., Chelsea, 1998.

[4] J. Kaczorowski and A. Perelli, The Selberg class: a survey, in: Number Theory in Progress, Proc. Conf. in Honor of A. Schinzel, K. Győry et al. (eds.), de Gruyter, 1999, 953-992.

[5] - - - On the structure of the Selberg class, $I: 0 \leq d \leq 1$, Acta Math. 182 (1999), 207-241.

[6] - - - On the structure of the Selberg class, II: invariants and conjectures, J. Reine Angew. Math. 524 (2000), 73-96.

[7] A. F. Leontiev, Exponential Series, Nauka, 1976 (in Russian).

[8] J. F. Ritt, On the zeros of exponential polynomials, Trans. Amer. Math. Soc. 31 (1929), 680-686.

[9] A. Shields, On quotients of exponential polynomials, Comm. Pure Appl. Math. 16 (1963), 27-31.

Faculty of Mathematics and Computer Science

A. Mickiewicz University

60-769 Poznań, Poland

E-mail: kjerzy@math.amu.edu.pl

Dipartimento di Matematica

Via Dodecaneso 35

16146 Genova, Italy

E-mail: perelli@dima.unige.it

Received on 12.10.2000

and in revised form on 29.11.2001 